\title{
Public health nurses used 4 strategies to facilitate client empowerment
}

\author{
Falk-Rafael AR. Empowerment as a process of evolving consciousness: a model of empowered caring. ANS Adv Nurs Sci \\ 2001 Sep;24:1-16.

\section{QUESTION: What is the practical meaning of empowerment to public health nurses and their clients?}

\begin{abstract}
Design
Exploratory qualitative design.

Setting

1 urban/rural and 2 rural health units in southwestern Ontario, Canada.

\section{Participants}

24 public health nurses $(100 \%$ women, mean 14.2 y in public health nursing) and 6 clients ( $83 \%$ women).

\section{Methods}

Nurses participated in a series of 3 two hour focus groups (5-9 participants per group; $71 \%$ participated in all 3 groups). Research questions were addressed using the nominal group technique. The researcher took field notes during the focus groups. Nurses identified clients whom they thought were empowered through their practice, and consenting clients were interviewed for about 1 hour using an interview guide. Interviews were audiotaped and transcribed. All data were coded and used to develop a model of empowerment.
\end{abstract}

\section{COMMENTARY}

The study by Falk-Rafael provides a global view of the concept of empowerment in health care, specifically related to the collaboration between clients and healthcare providers. The findings are consistent with other qualitative studies on empowerment. ${ }^{2}$ This study clearly presents a model of conceptualised empowerment through the narratives of public health nurses and their clients. The public health nurses identified empowerment in clients, which the clients verified. All clients reported increased assertiveness and a willingness to take control of their own health as a result of empowerment. This is consistent with "using the health promotion principle as 'client as expert'...".

Study findings are relevant to practice in clinical settings where nurses can empower their clients when setting mutually agreed upon goals. The nurse participants specifically mentioned a sense of dignity and a safe environment as critical to developing a trusting relationship between clients and nurses. These are key aspects of an effective learning environment in which the client and the nurse attempt to reach a mutual understanding of healthcare needs. This interaction represents a healthy trust rather than a blind trust. Empowered clients take more responsibility for their health and the study identifies this as "liberating". They have an increased sense of control, acquire and apply knowledge and skills, and effectively use resources.

When nurses are taught to empower clients, the clients will benefit as they acquire the positive assertiveness skills to take charge of their own health and wellbeing.

Debbie Hooser, RN, MSN

DNSc Student/Public Health University of Tennessee Health Sciences Center

Memphis, Tennessee, USA

1 Bartle EE, Couchonnal G, Canda ER, et al. Empowerment as a dynamically developing concept for practice: lessons learned from organizational ethnography. Soc Work 2002;47:32-43.

2 Pooley CG, Gerrard C, Hollis S, et al. 'Oh it's a wonderful practice...you can talk to them': a qualitative study of patients' and health professionals' views on the management of type 2 diabetes. Health Soc Care Community 2001;9:318-26.

3 Hutchings D. Partnership in education: an example of client and educator collaboration. J Contin Educ Nurs 1999;30:128-31.

\section{Main findings}

Nurses conceptualised empowerment as an active, internal process of growth that was rooted in personal or cultural beliefs, reached toward actualising one's full potential, and occurred within a nurturing nurse-client relationship. Nurses identified clients' active participation, increased awareness, and increased knowledge and skills as essential. Empowerment produced ripple effects that positively affected others.

Nurses facilitated empowerment using 4 strategies. Development of a trusting relationship required respect, empathy, and the creation of a safe environment. This relationship was characterised by mutuality, whereby nurses helped clients to identify health goals and negotiated their roles in attaining them. Clients identified the nurse's authenticity to be important. Advocacy included personal and political advocacy and was viewed as temporary in nature. Nurses linked clients to community resources and acted on their behalf to help them attain their health goals. Providing information and developing skills, from oral and written information to role modelling, allowed clients to make more informed choices and take more effective actions for their health goals, and to assert themselves with others. Capacity building, which involved reflective listening and an empathic approach, helped clients identify the resources or capacity to attain their goals. Nurses focused on strengths rather than limitations, and communicated an expectation of client accountability for actions and decisions.

Nurses identified 3 categories of outcomes of empowerment that were supported by clients' narratives. Changes in self included increased self confidence and self esteem. Changes in relationships with others resulted in improved relationships with family and friends and becoming more assertive with healthcare providers. Changes in behaviours occurred as clients made healthier choices for themselves and their families.

\section{Conclusions}

Public health nurses facilitated client empowerment using 4 strategies: development of a trusting relationship, advocacy, provision of information and developing skills, and capacity building. Outcomes of empowerment were changes in self, relationships with others, and behaviours. 